\title{
Oscillations in Cardiovascular Function During Acute Hypoxia in the Newborn Piglet Are Associated With Less Neurological Damage and Occur More Frequently in Females
}

\author{
THOMAS A. HARRIS, GENEVIEVE N. HEALY, PAUL B. COLDITZ, AND BARBARA E. LINGWOOD \\ Perinatal Research Centre [T.A.H., P.B.C., B.E.L.], University of Queensland Centre for Clinical Research, The University of Queensland, \\ Royal Brisbane and Women's Hospital, Queensland, Australia 4029; Cancer Prevention Research Centre School of Population Health \\ [G.N.H.], The University of Queensland, Queensland, Australia 4006
}

\begin{abstract}
The function of the cardiovascular system during hypoxia is an important determinant of neurologic outcome. Oscillations in blood pressure, particularly type- 3 waves with a duration of 10 to $160 \mathrm{~s}$, have been shown to occur in the presence of hypoxia in the neonatal rat. The aim of this study was to determine the characteristics and occurrence of type- 3 waves in the neonatal piglet and any relationship to neurologic damage after acute global hypoxia. Hypoxia/hypercarbia was induced in 32 anesthetized piglets by reducing the fraction of inspired oxygen to 0.1 and the ventilation rate from 30 to 10 breaths per minute for $45 \mathrm{~min}$. The degree of neurologic damage was assessed using both physiologic (EEG amplitude and cerebral impedance at $6 \mathrm{~h}$ posthypoxia) and structural (microtubule associated protein-2 immunohistochemistry) markers. Type- 3 waves in cardiovascular function occurred in 56\% of animals. An oscillating pattern was significantly associated with less neurologic damage $(p=0.01)$ and a lower duration of hypotension during hypoxia $(p=0.02)$, and occurred more frequently in females $(p=$ 0.024). (Pediatr Res 65: 504-508, 2009)
\end{abstract}

$\mathrm{P}$ erinatal asphyxia is a significant contributor to poor neurodevelopmental outcome and occurs in four in every 1000 live births (1). Our own data have shown that serum cortisol concentrations are also related to neurologic damage and cardiovascular function, an effect which may be mediated via the activity of the sympathetic nervous system (SNS) (2). Several studies have reported that the cardiovascular system can exhibit an oscillating response during periods of stress, such as hypoxia and cerebral ischemia (3-6). Oscillations in cardiovascular function occur at different frequencies. For example, type- 1 and -2 waves have a 5 -s cycle and result from oscillations in the vasomotor control mechanism, which involve both the baro- and chemoreceptor reflexes (3).

In comparison, type- 3 waves are slower with a cycle period of 10 to $160 \mathrm{~s}$. They have been observed in the presence of hypoxia, hemorrhage, cold, endotoxic shock, and increased intracranial pressure (4). Type-3 waves in blood pressure have been reported to occur in neonatal rats during acute hypoxia (5). The presence of these oscillations during hypoxia may be a protective mechanism against the subsequent neurologic injury after hypoxia/ischemia. Moorthy et al. (6) proposed that

Received July 30, 2008; accepted December 13, 2008.

Correspondence: Barbara Lingwood M.Sc., Ph.D., Perinatal Research Centre, UQ Center for Clinical Research, Royal Brisbane and Women's Hospital, Herston, Queensland 4029, Australia; e-mail b.lingwood@uq.edu.au when cerebral perfusion falls below a critical level, the development of cerebral ischemia triggers a feedback mechanism, resulting in an increase in blood pressure, heart rate (HR), and cardiac output thus increasing cerebral blood flow (CBF). Once blood flow to the brain has been restored, the mechanism switches off and consequently blood pressure and HR fall again if hypoxia continues. This rise and fall, which is the characteristic oscillating pattern, is considered to be dependent on the oxygen concentration (5). It is also likely that the activity of the SNS plays a role in the development of type-3 waves (7). These waves have not been widely studied, particularly in recent times, and the mechanisms and consequences of these oscillations have not been fully elucidated.

The aims of this study were to determine i) the characteristics and occurrence of type-3 waves in cardiovascular function during acute global hypoxia and ii) their possible relationship to the severity of neurologic damage in the neonatal piglet. This knowledge may lead to further understanding of the physiologic basis of the increased hypoxia tolerance observed in some individuals (2).

\section{METHODS AND MATERIALS}

Animals. Thirty-two 1-d-old piglets (Landrace $\times$ Large White) from 26 litters were studied. Control piglets $(n=5)$ underwent the same procedure with the exception of hypoxia and data from these animals were used in the calculation of the neurologic outcome score. This study was approved by the Animal Ethics Committee of the University Queensland and complies with the NHMRC Australian Code of Practice for the Care and Use of Animals for Scientific Purposes.

Surgical preparation. Surgical preparation has been previously described by Lingwood et al. (8). In brief, animals were initially anesthetized with 1 to 2\% halothane (Rhône Mérieux, Melbourne, Australia) in air. Anesthesia was maintained with propofol $(10 \mathrm{mg} / \mathrm{kg} / \mathrm{h}$; Diprivan, Zeneca, Macclesfield, United Kingdom) and alfentanil (55 $\mu \mathrm{g} / \mathrm{kg} / \mathrm{h}$; Rapifen, ICI Pharmaceuticals, Melbourne, Australia). Piglets were intubated (cuffed, size 3.0; Portex, Laboratorie Portex SA, Kent, United Kingdom) and ventilated (Newborn 250, SLE Ltd., Croydon, United Kingdom) at a rate of 30 breaths per minute (BPM), with ventilation adjusted to maintain $\mathrm{SaO}_{2} 95$ to $98 \%$ and $\mathrm{PaCO}_{2} 35$ to 45 $\mathrm{mm} \mathrm{Hg}$. Piglets were warmed using a radiant heater to maintain rectal temperature 38.5 to $39.5^{\circ} \mathrm{C}$. An umbilical artery catheter (3.5F; Sherwood Medical Company, St. Louis, MO) was inserted to monitor mean arterial pressure (MAP) and HR, and to collect blood samples. Hypotension was

Abbreviations: BPM, breaths per minute; CBF, cerebral blood flow; CFM, cerebral function monitoring; $\mathbf{F i O}_{\mathbf{2}}$, fraction of inspired oxygen; HR, heart rate; MAP, mean arterial pressure; MAP2, microtubule associated protein-2; SBF, skin blood flow; SNS, sympathetic nervous system 
defined as the time MAP was below $70 \%$ of baseline. Glucose $(10 \%)$ was delivered via a mammary vein at a rate of $3 \mathrm{~mL} / \mathrm{kg} / \mathrm{h}$. Two channels of electroencephagram (EEG) were recorded (Brainz Instruments, San Carlos, $\mathrm{CA})$. Cerebral function monitoring (CFM) was obtained from software within the instrument by converting these two channels of EEG to a single channel CFM output.

Skin and cerebral blood flow. Skin blood flow (SBF) and CBF were measured continuously using laser Doppler flowmetry (ADInstruments, Sydney, Australia). The skin flow probe was positioned just above the thigh, after shaving and cleaning the skin. The cerebral probe $(1 \mathrm{~mm}$ diameter $)$ was inserted $0.1 \mathrm{~cm}$ into the parasaggittal cortex through a 3-mm burr hole in the skull.

Blood sampling. Arterial blood gases (Radiometer Pacific, ABL 520, Copenhagen, Denmark) and glucose were assessed before hypoxia and at 10-min intervals during hypoxia. The serum concentration of cortisol was determined using a competitive binding immunoenzymatic assay (ADVIA Centaur, Ireland) and measured before and at the end of hypoxia. The interassay variability $(\mathrm{CV})$ for cortisol was $6 \%$ at $125 \mathrm{nmol} / \mathrm{L}, 4.6 \%$ at 564 $\mathrm{nmol} / \mathrm{L}$, and $4.8 \%$ at $880 \mathrm{nmol} / \mathrm{L}$; the intraassay variability was $4.7 \%$ at 137 $\mathrm{nmol} / \mathrm{L}, 4.9 \%$ at $558 \mathrm{nmol} / \mathrm{L}$, and $5.5 \%$ at $884 \mathrm{nmol} / \mathrm{L}$.

Hypoxia. The hypoxic/hypercarbic insult was designed to mimic as closely as possible the human situation that includes mixed metabolic and respiratory acidosis. Thus, hypoxia was global, and ischemia occurred not as a result of carotid occlusion, but because of the progressive failure of cardiovascular function as the heart itself became affected by hypoxia. Ventilation rate, as well as the fraction of inspired oxygen $\left(\mathrm{FiO}_{2}\right)$, was reduced to mimic reduced clearance of $\mathrm{CO}_{2}$ that would occur in the human neonate experiencing cord occlusion or placental separation. The duration of hypoxia chosen, produces a variety of outcomes ranging from little or no neurologic damage to severe injury. In this model, injury occurs in the same brain regions susceptible to hypoxic injury in the human infant (9). Resuscitation procedures were also based on the commonly used clinical protocols at the time of the experiments.

Pancuronium (0.2 mg/kg; Astra Zeneca, Sydney, Australia) was given 5 min before hypoxia to prevent spontaneous respiration. Global hypoxia was induced by reducing the $\mathrm{FiO}_{2}$ to 0.1 and decreasing the ventilation rate from 30 to $10 \mathrm{BPM}$ for $45 \mathrm{~min}$. Hypoxia was terminated by rapidly increasing the $\mathrm{FiO}_{2}$ to 1.0 and returning the ventilation rate to $30 \mathrm{BPM}$. $\mathrm{FiO}_{2}$ was decreased within 15 min to the lowest value consistent with $\mathrm{SaO}_{2}>94 \%$. Six hours after hypoxia, the animal was euthanized using 3-mL Lethobarb (sodium pentobarbitone $325 \mathrm{mg} / \mathrm{mL}$, i.v.) and the brain was removed for immunohistochemical analysis (10).

Determination of neurologic damage. The severity of neurologic damage was determined using a combination of physiologic and structural markers as previously described by Harris et al. (2). Physiologic markers used were maximum amplitude of EEG, and cerebral impedance. These markers are closely correlated with longer term histologic damage (8), and our group has observed a close correlation between histologic damage and functional outcomes such as motor control in the piglet (unpublished data). Microtubuleassociated protein-2 (MAP2) immunohistochemistry was used as a structural marker at $6 \mathrm{~h}$ post hypoxia. MAP2 immunoreactivity is proportional to the severity of brain injury and allows comparison of injury between animals (10). Each of these outcome measures was converted to a score of 10 , which measured injury with reference to control animals. These were combined using the formula below to give a final outcome score (with $0=$ poor outcome and $20=$ good outcome) giving equal weighting to physiology and structure.

Neurologic damage score $=[$ (impedance score + EEG score $) / 2]+$ standardized MAP2 score (2).

Analysis of oscillations in cardiovascular function. Animals were divided into two groups based on the presence or absence of oscillations. There was no need to define further criteria for entry to the oscillation group as oscillations were clearly either present or absent on the trace (Fig. 1). The oscillating pattern in MAP and HR was characterized by measuring the height of each peak compared with the MAP or HR immediately before each oscillation. The duration of each oscillation was based on trough-to-trough time.

Statistics analysis. Data are expressed as mean \pm SD. Two-way ANOVA with Bonferroni post hoc analysis was used to compare variables measured at multiple time points between oscillating and nonoscillating groups (including arterial $\mathrm{pH}, \mathrm{PaCO}_{2}$, and glucose). $t$ test was used to compare variables measured at a single time point (duration and depth of anesthesia, serum cortisol concentrations, duration of hypotension, time HR was below baseline, and neurologic damage score) between oscillating and nonoscillating groups. Pearson $\chi^{2}$ test was used to determine gender differences between the oscillating and nonoscillating groups (Stata for Windows; Version 8, StataCorp LP, College Station, TX). A value of $p<0.05$ was considered significant.
A
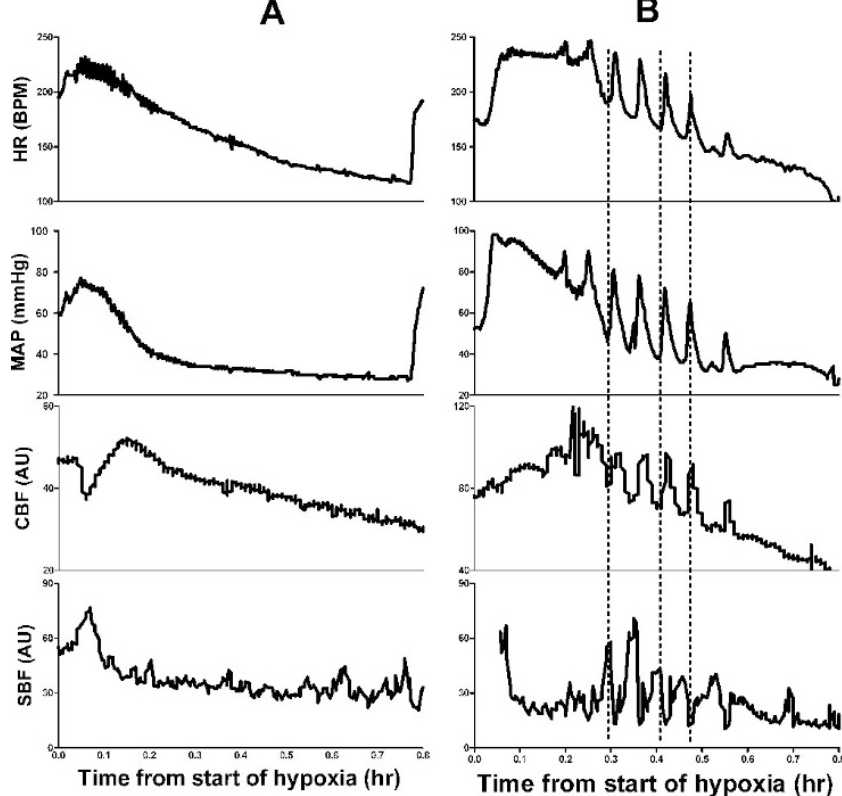

Figure 1. Example of a nonoscillating (panel $A$ ) and oscillating (panel B) response during hypoxia. $\mathrm{HR}$, heart rate; MAP, mean arterial pressure; $\mathrm{CBF}$, cerebral blood flow; SBF, skin blood flow; AU, arbitary units. Broken lines have been inserted on some cycles to illustrate the parallel or opposing nature of the changes.

\section{RESULTS}

The mean $( \pm \mathrm{SD})$ weight of the 32 piglets studied was $1.5 \pm$ $0.2 \mathrm{~kg}$. There were $18(61 \%)$ males and $14(39 \%)$ females from litters ranging in size from 6 to 19 piglets per litter. Eighteen (56\%) animals exhibited oscillation in cardiovascular function. In most cases (78\%), the oscillation pattern continued until resuscitation. No oscillations were observed in control animals $(n=5)$.

Females exhibited oscillations more frequently than males. Eleven $(79 \%)$ of the female piglets exhibited an oscillating pattern in cardiovascular function while oscillations occurred in only seven (39\%) of the male piglets $(\chi=5.0, p=0.024)$. There was no difference between male and female piglets in any baseline variables, cardiovascular function or cortisol levels during hypoxia, or the severity of neurologic damage. There was no difference between oscillating and nonoscillating groups in baseline cardiovascular or hormonal variables. Weight $(p=0.21)$ and age $(p=0.78)$ were not significantly different between the oscillating and nonoscillating groups. There was no significant difference in the duration of anesthesia ( $p=0.15$ ) or the depth of anesthesia as evident by CFM amplitude $(p=0.11)$ before hypoxia between the oscillating and nonoscillating groups.

Figure 1 shows a typical example of an oscillating pattern and a nonoscillating pattern. In all oscillating cases MAP, HR, and $\mathrm{CBF}$ moved in parallel whereas SBF moved in the opposite direction. A wide range of values for MAP (32 to 77 $\mathrm{mm} \mathrm{Hg}$ ) and percentage change in CBF (73 to 203\%) was observed before the onset of oscillation. The features of the oscillations in cardiovascular function are listed in Table 1. The number of oscillations observed in each animal in the oscillating group ranged from 2 to 13 . 
Table 1. Characteristics of oscillations during hypoxia. Data represent the mean values for all animals in the oscillating group $(\mathrm{n}=18)$

\begin{tabular}{lc}
\hline \multicolumn{1}{c}{ Features of oscillations } & Mean \pm SD \\
\hline Time after onset of hypoxia that oscillation & $23.2 \pm 8.3$ \\
$\quad$ commenced (min) & \\
Amplitude of oscillations in MAP $(\mathrm{mm} \mathrm{Hg})$ & $22.5 \pm 9.8$ \\
Period of oscillation (min) & $2.6 \pm 0.25$ \\
MAP before onset of oscillation $(\mathrm{mm} \mathrm{Hg})$ & $50.5 \pm 13.0$ \\
CBF prior to onset of oscillation $(\%$ change & $107.7 \pm 40.7$ \\
$\quad$ from baseline) & \\
CFM at onset of oscillation $(\mu \mathrm{V})$ & $6.0 \pm 3.0$ \\
\hline
\end{tabular}

Table 2 reports the physiologic characteristics during hypoxia and the neurologic damage score for the oscillating and nonoscillating groups. ANOVA revealed that there was no difference between the oscillating and nonoscillating groups in arterial $\mathrm{pH}, \mathrm{PaO}_{2}$, or glucose at any time point during hypoxia. The duration of hypotension during hypoxia, and arterial $\mathrm{PaCO}_{2}$ concentrations at the end of hypoxia were significantly lower in the oscillating group compared with the nonoscillating group, whereas serum cortisol concentrations were significantly higher in the oscillating group compared with the nonoscillating group (Table 2). All other physiologic variables were not significantly different between the two groups. The individual neurologic damage scores for all animals in the oscillating and nonoscillating groups are shown in Figure 2. Neurologic outcome scores were significantly higher in the oscillating group (Table 2).

\section{DISCUSSION}

To our knowledge, this is the first study to demonstrate that an oscillating pattern in MAP, HR, CBF, and SBF in the

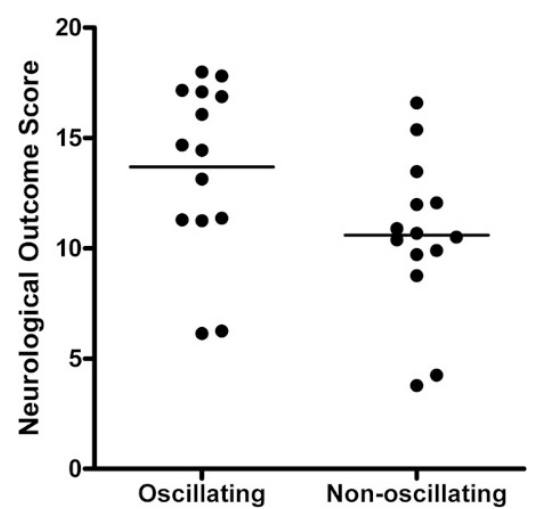

Figure 2. Neurologic outcome scores in individual animals for the oscillating and nonoscillating animals. Neurologic outcome scores for individual animals exhibiting an oscillating or nonoscillating pattern. Line represents mean values.

neonatal piglet during acute global hypoxia is associated with less neurologic damage after hypoxia. Overall, 56\% of animals exhibited an oscillating pattern in MAP, $\mathrm{HR}, \mathrm{CBF}$, and SBF matching the definition of type-3 waves based on the mean period of their oscillation (156 s). This corresponds to a frequency of $<0.01 \mathrm{~Hz}$, lower than even very low frequency waves often reported (11). An oscillating pattern was associated with significantly lower duration of hypotension and higher serum cortisol concentration compared with the nonoscillating group. Male piglets exhibited oscillating cardiovascular function less frequently than females. There was no significant difference in neurologic damage between genders, possibly due to the high degree of variability in damage scores and limited statistical power. Another study has suggested that

Table 2. Comparison of physiological variables between animals that exhibited an oscillating (Osc) mean arterial pressure ( $\mathrm{n}=18)$ and those that did not exhibit an oscillating pattern (Nonosc) $(\mathrm{n}=14)($ mean $\pm S D)$ during acute global hypoxia

\begin{tabular}{|c|c|c|c|c|c|}
\hline Variable & Baseline & $10 \mathrm{~min}$ & $20 \mathrm{~min}$ & $30 \mathrm{~min}$ & End \\
\hline \multicolumn{6}{|l|}{ Arterial $\mathrm{pH}$} \\
\hline Osc & $7.44 \pm 0.05$ & $7.17 \pm 0.04$ & $7.07 \pm 0.08$ & $6.95 \pm 0.07$ & $6.80 \pm 0.11$ \\
\hline Nonosc & $7.47 \pm 0.05$ & $7.17 \pm 0.06$ & $7.05 \pm 0.07$ & $6.92 \pm 0.07$ & $6.77 \pm 0.08$ \\
\hline \multicolumn{6}{|l|}{$\mathrm{PaO}_{2}(\mathrm{~mm} \mathrm{Hg})$} \\
\hline Osc & $90.9 \pm 13.7 *$ & $19.8 \pm 3.7$ & $21.5 \pm 3.7$ & $24.3 \pm 4.0$ & $25.5 \pm 2.9$ \\
\hline Nonosc & $100.0 \pm 13.5$ & $21.2 \pm 6.6$ & $22.0 \pm 4.1$ & $22.9 \pm 4.6$ & $25.6 \pm 5.4$ \\
\hline \multicolumn{6}{|l|}{$\mathrm{PaCO}_{2}(\mathrm{~mm} \mathrm{Hg})$} \\
\hline Osc & $40.3 \pm 3.4$ & $60.9 \pm 5.1$ & $65.6 \pm 6.5$ & $73.0 \pm 7.5$ & $80.3 \pm 11.6^{*}$ \\
\hline Nonosc & $40.0 \pm 4.5$ & $62.9 \pm 5.4$ & $72.7 \pm 11.9$ & $83.8 \pm 15.1$ & $95.2 \pm 21.5$ \\
\hline \multicolumn{6}{|l|}{ Glucose (mmol/L) } \\
\hline Osc & $4.8 \pm 1.1$ & $6.6 \pm 1.7$ & $7.5 \pm 2.6$ & $7.7 \pm 2.8$ & $7.9 \pm 4.0$ \\
\hline Nonosc & $4.8 \pm 0.9$ & $6.7 \pm 1.8$ & $7.5 \pm 2.4$ & $8.2 \pm 3.2$ & $8.9 \pm 4.2$ \\
\hline \multicolumn{6}{|l|}{ Cortisol (nmol/L) } \\
\hline Osc & $127 \pm 55$ & NA & NA & NA & $384 \pm 207 *$ \\
\hline Nonosc & $149 \pm 62$ & NA & NA & NA & $218 \pm 132$ \\
\hline \multicolumn{6}{|c|}{ Duration of hypotension (time MAP is below $70 \%$ baseline) (min) } \\
\hline Osc & NA & NA & NA & NA & $9.3 \pm 9.3^{*}$ \\
\hline Nonosc & NA & NA & NA & NA & $23.5 \pm 12.0$ \\
\hline \multicolumn{6}{|l|}{ Time HR is below baseline ( $\mathrm{min}$ ) } \\
\hline Osc & NA & NA & NA & NA & $9.1 \pm 10.5$ \\
\hline Nonosc & NA & NA & NA & NA & $14.9 \pm 12.9$ \\
\hline \multicolumn{6}{|l|}{ Neurologic outcome $\dagger$} \\
\hline Osc & NA & NA & NA & NA & $13.7 \pm 4.0^{*}$ \\
\hline Nonosc & NA & NA & NA & NA & $10.6 \pm 3.6$ \\
\hline
\end{tabular}

* Variables significantly different $(p<0.05)$ between oscillating and nonoscillating cardiovascular function groups.

$\dagger$ Four of 18 animals in the oscillating group did not have neurologic outcome assessment as they were euthanized directly after hypoxia for other assessments. 
detection of gender differences requires large numbers $(>100)$ of animals with differences only apparent in specific subgroups (12).

Overall, animals in the oscillating group had less neurologic damage compared with animals in the nonoscillating group (Table 2, Fig. 2). It is of interest that two animals in the oscillating group had a poor outcome. These two animals still exhibited a long duration of hypotension ( 23 to $33 \mathrm{~min}$ ) during hypoxia regardless of the presence of oscillations. Furthermore, it can be seen that some animals in the nonoscillating group had a good neurologic outcome. These animals were able to maintain cardiovascular function effectively despite the absence of oscillations and had very short durations of hypotension ( 0 to $9 \mathrm{~min}$ ) during hypoxia. The association between the occurrence of oscillations and the reduced duration of hypotension is probably responsible for the overall improved outcome in the group that exhibited oscillations (2). A key feature of the oscillating pattern was the periodic increases in MAP. Blood pressure is a critical factor in determining neurologic outcome because autoregulation is impaired during hypoxia and thus the cerebral circulation becomes pressure passive (13-18). We propose that these oscillations in cardiovascular function restore blood flow to the brain and remove harmful metabolites $\left(\mathrm{H}^{+}\right.$and $\left.\mathrm{CO}_{2}\right)$. This may also account for lower $\mathrm{PaCO}_{2}$ concentrations at each time point in the oscillating group. Improved blood flow through the lungs during the periods of increased blood pressure may lead to more effective clearance of $\mathrm{CO}_{2}$.

The mechanisms involved in the commencement of oscillation in cardiovascular function were not assessed in the current study; however, a number of observations can be made. Dora et al. (5) suggested that $\mathrm{PaO}_{2}$ was a key mechanism for the commencement of oscillations. We found that at all time points during hypoxia, $\mathrm{PaO}_{2}$ concentrations were not different in the oscillating and nonoscillating groups and thus were not able to confirm that $\mathrm{PaO}_{2}$ is the factor triggering the commencement of oscillation. The range of values observed for MAP immediately before oscillations commenced (32 to $77 \mathrm{~mm} \mathrm{Hg}$ ) was very variable as were the CBF and CFM amplitudes. Thus, there does not appear to be a consistent level of MAP or CBF at which the oscillating mechanism is triggered. Likewise, the degree of brain activity (CFM amplitude) does not seem to influence the commencement of oscillations in cardiovascular function. Perhaps reduced cerebral oxygen delivery or build up of hypoxia related metabolites in the brain (rather than hypoxia or ischemia alone) are responsible for triggering this phenomenon.

Previous studies in adult animals show that the SNS is involved in the commencement of oscillation. Guyton and Statterfield (7) and Moorthy et al. (6) demonstrated that cerebral ischemia causes increased activity of the SNS resulting in oscillation in cardiovascular function. Lang et al. (19) showed that the number and amplitude of type-3 waves was reduced in comatose adult patients receiving SNS antagonists compared with healthy controls. These studies in the adult indicate that the SNS plays an important role in determining whether oscillations in cardiovascular function will occur. We propose that a triggering mechanism such as decreased cere- bral oxygen delivery results in increased SNS activity increasing blood pressure and $\mathrm{HR}$ and peripheral vasoconstriction. As cerebral oxygen delivery is restored, SNS activity is reduced, and blood pressure and HR fall again as a result of continued hypoxia. The process is repeated as the response is triggered a second time. An alternative explanation is that the build up of metabolites in the periphery results in an inability to maintain peripheral vasoconstriction thus vasodilation occurs and blood pressure falls. However, if this were the case HR would either be unaltered or would increase as the baroreflex responds to falling blood pressure. This is not the case as HR oscillates in parallel with blood pressure. The failure of some animals to exhibit this oscillating response may be due to insensitivity to the triggering event or immaturity of the SNS responses.

Interestingly, in the current study, serum cortisol concentrations were significantly higher in the oscillating group compared with the nonoscillating group. It is well known that cortisol has permissive effects on the cardiovascular system by enhancing the vascular effects of catecholamines (20-22), which may play a crucial role in the development of oscillations. In addition to the vascular effects of cortisol, higher cortisol levels in the neonate reflect increased SNS activity/ maturity, an effect that occurs more frequently in females (23-25). Thus, higher cortisol concentrations in the current study may point to greater activity of the SNS during hypoxia, which may contribute to the commencement of oscillations in cardiovascular function. Future studies should include assessment of SNS activity.

Males exhibited oscillation less frequently than females and are thus at greater risk of neurologic damage. This supports the suggestion by other studies that the SNS and compensatory mechanisms to hypoxia are more mature in females than males (12,25-27). Padbury et al. (25) demonstrated that the maturation and function of autonomic nervous system is delayed in male rabbits compared with females. In addition, preterm male infants have lower plasma catecholamine levels than females after birth asphyxia (28). It has also been shown that when both genders are exposed to progressively lower oxygen concentrations males died earlier than females, suggesting that females are more resistant to hypoxia than males (26). We postulate that decreased maturity of the SNS in some males results in an inability to initiate an appropriate cardiovascular responses to hypoxia, and inability to maintain adequate cardiovascular function in the face of hypoxia. This in turn leads to inadequate $\mathrm{CBF}$ increasing the risk of neurologic injury. This area warrants further investigation as it may explain why males are at greater risk of perinatal brain injury and later neurologic and cognitive impairment after hypoxia/ ischemia (23-26).

A limitation of this study was the use of anesthesia in this model of perinatal asphyxia. Although anesthesia could potentially affect cardiovascular function and severity of neurologic damage, the anesthetic regime was the same for each animal and thus these effects will be constant across all animals. The duration, dose rate, or depth of anesthesia before or during hypoxia was not related to the occurrence of oscillations. In addition, neurologic damage was not able to be 
assessed in four of 18 animals in the oscillating group. However, duration of hypotension in these animals was minimal ( 0 to $8.1 \mathrm{~min}$ ), and thus it is likely that their outcome scores would have been very high increasing the difference between the oscillating and the nonoscillating groups.

In conclusion, the development of type- 3 waves during acute global hypoxia occur more frequently in females and are associated with reduced neurologic damage in the neonatal piglet. This study supports other evidence that the maturity of the SNS may be responsible for the better outcomes observed in female babies admitted to Neonatal Intensive Care Units.

\section{REFERENCES}

1. Vannucci RC, Perlman JM 1997 Interventions for perinatal hypoxic-ischemic encephalopathy. Pediatrics 100:1004-1014

2. Harris TA, Healy GN, Colditz PB, Lingwood BE 2008 Association between serum cortisol, cardiovascular function and neurological outcome following acute global hypoxia in the newborn piglet. Stress Oct 24:1 [Epub ahead of print]

3. Guyton AC, Harris JW 1951 Pressoreceptor-autonomic oscillation; a probable cause of vasomotor waves. Am J Physiol 165:158-166

4. Seiver A, Daane S 1996 Regular low frequency cardiac output oscillations observed in critically ill surgical patients. Complexity 2:51-55

5. Dora E, Olaffson K, Chance B, Kovach AG 1976 Cortical NADH, pO2, electrical activity and arterial blood pressure oscillations in hypoxaemia. Adv Exp Med Biol 75:299-305

6. Moorthy SS, Stoelting RK, King RD 1983 Delayed cyclic variations (oscillations) in blood pressure in a critically ill patient. Crit Care Med 11:476-477

7. Guyton AC, Statterfield JH 1952 Vasomotor waves possibly resulting from CNS ischemic reflex oscillation. Am J Physiol 170:601-605

8. Lingwood BE, Dunster KR, Healy GN, Ward LC, Colditz PB 2003 Cerebral impedance and neurological outcome following a mild or severe hypoxic/ischemic episode in neonatal piglets. Brain Res 969:160-167

9. Bjorkman ST, Foster KA, O’Driscoll SM, Healy GN, Lingwood BE, Burke C, Colditz PB 2006 Hypoxic/ischemic models in newborn piglet: comparison of constant $\mathrm{FiO}_{2}$ versus variable $\mathrm{FiO}_{2}$ delivery. Brain Res 1100:110-117

10. Lingwood BE, Healy GN, Sullivan SM, Pow DV, Colditz PB 2008 MAP2 provides reliable early assessment of neural injury in the newborn piglet model of birth asphyxia. J Neurosci Methods 171:141-146
11. Radaelli A, Castiglioni P, Centola M, Cesana F, Balestri G, Ferrari AU, Di Rienzo M 2006 Adrenergic origin of very low-frequency blood pressure oscillations in the unanesthetized rat. Am J Physiol Heart Circ Physiol 290:H357-H364

12. Bennet L, Booth LC, Ahmed-Nasef N, Dean JM, Davidson J, Quaedackers JS, Gunn AJ 2007 Male disadvantage? Fetal sex and cardiovascular responses to asphyxia in preterm fetal sheep. Am J Physiol Regul Integr Comp Physiol 293:R1280-R1286

13. Tweed WA, Cote J, Pash M, Lou H 1983 Arterial oxygenation determines autoregulation of cerebral blood flow in the fetal lamb. Pediatr Res 17:246-249

14. Bender TM, Johnston JA, Manepalli AN, Mink RB 2003 Association between brain tissue $\mathrm{pH}$ and brain injury during asphyxia in piglets. Resuscitation 59:243-254

15. Mallard EC, Gunn AJ, Williams CE, Johnston BM, Gluckman PD 1992 Transient umbilical cord occlusion causes hippocampal damage in the fetal sheep. Am J Obstet Gynecol 167:1423-1430

16. Williams CE, Mallard C, Tan W, Gluckman PD 1993 Pathophysiology of perinatal asphyxia. Clin Perinatol 20:305-325

17. de Courten-Myers GM, Fogelson HM, Kleinholz M, Myers RE 1989 Hypoxic brain and heart injury thresholds in piglets. Biomed Biochim Acta 48:S143-S148

18. Low JA 2004 Determining the contribution of asphyxia to brain damage in the neonate. J Obstet Gynaecol Res 30:276-286

19. Lang EW, Diehl RR, Timmermann L, Baron R, Deuschl G, Mehdorn HM, Zunker P 1999 Spontaneous oscillations of arterial blood pressure, cerebral and peripheral blood flow in healthy and comatose subjects. Neurol Res 21:665-669

20. Yang S, Zhang L 2004 Glucocorticoids and vascular reactivity. Curr Vasc Pharmacol 2:1-12

21. Hanson MA 1997 Do we now understand the control of the fetal circulation? Eur J Obstet Gynecol Reprod Biol 75:55-61

22. Giussani DA, Spencer JA, Hanson MA 1994 Fetal cardiovascular reflex responses to hypoxaemia. Fetal Matern Med Rev 6:17-37

23. Lee JC, Werner JC, Downing SE 1980 Adrenal contribution to cardiac responses elicited by acute hypoxia in piglets. Am J Physiol 239:H751-H755

24. Ehrhart-Bornstein M, Hinson JP, Bornstein SR, Scherbaum WA, Vinson GP 1998 Intraadrenal interactions in the regulation of adrenocortical steroidogenesis. Endocr Rev 19:101-143

25. Padbury JF, Hobel CJ, Lam RW, Fisher DA 1981 Sex differences in lung and adrenal neurosympathetic development in rabbits. Am J Obstet Gynecol 141:199-204

26. Mage DT, Donner M 2006 Female resistance to hypoxia: does it explain the sex difference in mortality rates? J Womens Health (Larchmt) 15:786-794

27. Hindmarsh GJ, O'Callaghan MJ, Mohay HA, Rogers YM 2000 Gender differences in cognitive abilities at 2 years in ELBW infants. Extremely low birth weight. Early Hum Dev 60:115-122

28. Greenough A, Lagercrantz H, Pool J, Dahlin I 1987 Plasma catecholamine levels in preterm infants. Effect of birth asphyxia and Apgar score. Acta Paediatr Scand 76:54-59 